\title{
EDITORIAL
}

\section{Mobile computing}

Technology in recent years has developed very fast, due to advances in materials technology and microelectronics, reason for which the beneficiary areas include communications, computing and information. Currently in technologically developed countries, such as Japan and the United States among others, mobile communication has increased, whereas fixed communication presents a setback. With regard to Latin America, on average, the number of mobile phone users has grown by $90 \%$ in the period of the last 10 years. Likewise, a similar trend shows the use of computers and the Internet both for home, business and industry.

Trends in the use of mobile communications technology have several points of view, such that "technology makes possible and facilitates certain types of user behavior" or that "the successful incorporation of technology in society, encourages phases and predictable steps". Whatever the view, the mobile communication technology has been supplemented by computer systems. Mobile communication initially consisted of oral communication, and currently has the capacity for group work distance: instant messaging, information sharing and its modification by different applications and other features.

Some studies in the social-industrial and services indicate that the key factors are communication productivity and mobility of staff, which includes oral and written communication and capability to communicate information in different formats and a solution to use these factors are the computational and communication systems for mobile devices. The development of these studies and technology has led to the creation of new research areas such as:

Software: Operating systems, multimedia on mobile devices, mobility modeling, application programming for mobile devices, development of paradigms for heterogeneous applications, mobile database, artificial intelligence-oriented mobility and exchange of information, mobility software engineering, and wireless networks, their protocols and security.

Hardware: Architectures for low resource devices, display technologies, circuit bundles, special-purpose circuits, energy saving and battery technology.

Education: Multimedia-oriented education, mobile education models, learning methodologies incorporating the resources of mobile devices and the ubiquity of information and applications

Human-Computer Interaction: Plasticity and adaptability, use of multimodal interfaces, management of mobile device sensors, metrics for human computer interaction (usability, functionality and performance), perceptual information, ergonomics, 3D management and development of 3D immersion Mobile mechanism.

Communication: Global Wireless networks as GSM (Global System for Mobile communications), 3G and 4G (3rd and 4th generation mobile digital communications networks), security protocols and architecture for voice and data transmission. Integration of various technologies of wireless and wired communications.

These and other areas of study together manipulate, process, store or transmit data, along with technological advances and wireless and mobile communication is known today as mobile computing technology. 
Mobile computing, as an emerging discipline in computing, marks a future trend towards "Telecommuting" or "e-work", which is work remotely performed with the use of mobile devices, computer systems and the Internet. Users of mobile devices increase annually, giving rise to the rapidly evolving mobile service, and requiring new hardware technologies, both low resources and high efficiency and software.

The vision of enterprise mobility and services is being taken into account by large corporations, who invest a large capital in the development of electronic components and software. On the other hand, universities and institutions have to generate corresponding human resources able to implement innovate and develop new mobile technology aimed at applications such as collaborative work, automation and industrial control, remote education, and entertainment among others.

A documented case, where the future is investing in mobile communication device is the one of iPhone, which is estimated that over 50\% of shoppers planning to buy a smart phone to bow to it. One factor that has contributed to the iPhone phenomenon has to do with the software package developed for this device. Importantly, the market competition has led other companies to provide mobile solutions, for example, Google offers a mobile platform (operating system) called Android, and Microsoft is making available new versions of Windows Mobile. The ease with which these devices and mobile technologies have flooded the market, has led to an increase of fifty times the access to internet by wireless means in the last three years, according to studies published in the 13th ACIS International Conference on Software Engineering, Artificial Intelligence, Networking and Parallel / Distributed Computing 2012, Kyoto, Japan.

The technology behind mobile computing devices is changing rapidly. A comprehensive review of all suppliers, services and applications targeted towards mobile computing is beyond the scope of this document, suffice to say that companies like Intel estimated that, in 2011 processors that reduce energy consumption to one-tenth of what current processors require were introduced in the market, beating one of the major limitations that currently show mobile computing devices.

From the foregoing it may be noted that:

- Mobile computing is becoming every day a technological paradigm most commonly used.

- Mobile computing will change the way work activities are carried out, academic, research and entertainment, as at the computer did at its time.

- Organizations of all kinds will have to migrate to electronic services to wireless service.

- The labor market will require researchers and professionals to address and resolve the challenges posed by mobile computing.

With regard to human resources who perform research, development and implementation of new technologies in mobile computing or any other new technology, are required to have a solid academic foundation, and having multidisciplinary training in the field of knowledge, human resources and graduate specialization are becoming more valued by companies, and technologies are what make the requirement of academic characteristics that must have the professional.

Jesús Yaljá Montiel Pérez $\begin{gathered}\text { Erika Hernández Rubio } \\ \text { Escuela Superior de Cómputo } \\ \text { Instituto Politécnico Nacional } \\ \text { México, D.F. }\end{gathered}$
E-mail: yalja@ipn.mx; ehernandezru@ipn.mx; jlopezb@ipn.mx

